\title{
Use of erlotinib throughout pregnancy: a case-report of a patient with metastatic lung adenocarcinoma
}

- Giovanna Rivas ${ }^{1,2}$, Néstor Llinás ${ }^{1,2}$, Carlos Bonilla ${ }^{1,2}$, Juan Rubiano ${ }^{1,2}$, Javier Cuello ${ }^{1,2}$, Natalia Arango $0^{1,2}$

${ }^{1}$ Clinical Oncology Group, Cancerology National Institute, ESE, Bogotá (Colombia).

2 Universidad El Bosque, Bogotá (Colombia).

The use of erlotinib throughout pregnancy has not been previously reported. We present the case of a 40 yearold female patient with stage IV lung adenocarcinoma, mediastinal, bone and cerebral metastasis, a EGFR mutation and no smoking history, who had begun first line treatment with erlotinib $150 \mathrm{mg}$ once daily. After two and a half months of treatment a fourteen-week pregnancy was documented, and after informing on fetal risks secondary to erlotinib use and maternal risks secondary to treatment withholding, she decided to continue with treatment under clinical surveillance by both the oncology and obstetrics clinics. At thirty-three weeks gestation a live born $1600 \mathrm{~g}$ female was born by caesarean section without evidence of congenital malformations. Imaging assessment after eight months of treatment showed complete bone and central nervous system response and partial lung and mediastinal response. The patient is currently undergoing the 11 th month of treatment and is asymptomatic, the baby is 4 months old and is in good health.

\section{Toxicidad severa de la piel secundaria al uso de erlotinib}

- Miguel Ángel Saavedra Hurtado, Álvaro J. Guerrero Villota, Juan Manuel Herrera, Diego López, Camilo Zorrilla, Indira Hernández, Margarita Velazco, Fernando Huertas, Henry Idrobo, Álvaro Gómez, Rigoberto Gómez, Yorlani Rodas

Objetivo. Describir un caso clínico real de toxicidad severa, grado IV, secundario a erlotinib de presentación poco frecuente.

Metodología. Paciente de 29 años de edad con diagnóstico de cáncer de pulmón, con mutación del gen del receptor de crecimiento epidérmico tipo 1, el cual recibió tratamiento con medicamento erlotinib, obteniendo excelente respuesta y control del tumor, pero con gran toxicidad dérmica que se extendía a cara, tronco, extremidades inferiores, superiores y espalda de manera simultánea.

Resultados y conclusiones. El paciente presentó notoria mejoría clínica de la toxicidad con la suspensión del medicamento y posterior reajuste de dosis; ocho meses después continúa con enfermedad estable y resolución de toxicidad dérmica.

La terapia molecular es una de las más recientes y novedosas alternativas en el tratamiento de enfermedades oncológicas, entre ellas la inhibición de los receptores de tirosina quinasa (IrTK).

La toxicidad dermatológica es la complicación más frecuente del uso de los medicamento inhibidores de la tirosina quinasa como el erlotinib, siendo causa importante de disminución de la dosis y/o retiro del tratamiento.

La revisión de la literatura reporta escasos casos de toxicidad severa que se extienden de manera simultánea a extremidades, tronco y cara; además, la clasificación actual de la toxicidad dérmica no tiene la suficiente precisión y objetividad para una adecuada categorización, lo cual es evidente en el caso presentado. Esto hace necesario profundizar en la elaboración de mejores estándares de clasificación para las toxicidades de IrTK. 